\title{
Endometrioid Borderline Tumor Related to Ovarian Endometriosis
}

\author{
Anna Protasova ${ }^{1 *}$, Anna Tsypurdeeva ${ }^{1,2}$, Grigory Raskin ${ }^{1,3}$ and Nurana Bairamova ${ }^{1}$ \\ ${ }^{1}$ Department of Medicine, St. Petersburg University, St. Petersburg, Russia \\ ${ }^{2}$ Ott Research Institute of Obstetrics, Gynecology and Reproductology, St. Petersburg, Russia \\ ${ }^{3}$ Department of Pathology, Russian Scientific Center for Radiology and Surgical Technologies, St. Petersburg, Russia \\ *Corresponding author: Anna Protasova, Department of Medicine, St. Petersburg University, St. Petersburg, Russia

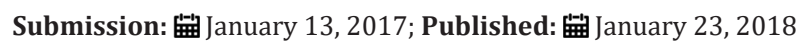

\section{Context}

Endometrioid borderline tumor (EBT), also known as atypical proliferative endometrioid tumor, accounts for $0.2 \%$ of ovarian neoplasmas and $2-3 \%$ of borderline ovarian tumors. Very little is known about the prognosis and management of this entity. It is important to correctly diagnose these rare tumors in young women to assure proper treatment and to prevent mortality and preserve fertility.

\section{Objective}

We report a case of EBT with endometriosis in a 26-year-old woman.

\section{Case Report}

A 26-year-old female patient diagnosed with a left ovarian mass was referred to our institution for elective surgery. The patient presented with chronic pelvic pain (pain score 60-70 $\mathrm{mm}$ on a 100-mm visual analog scale) and dysmenorrhea since menarche at the age of 14 years, requiring the intake of nonsteroidal antiinflammatory drugs and leading to asthenia. Ovarian mass was

Result

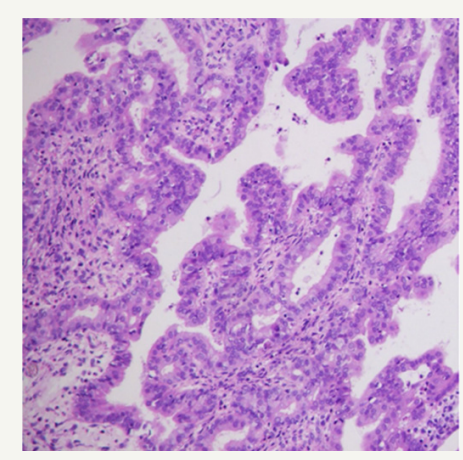

(a)

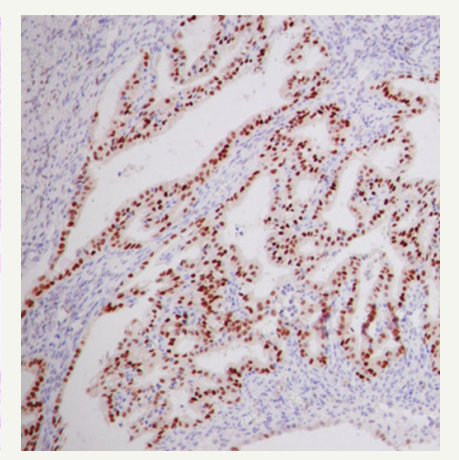

(b) found 3 years earlier by ultrasonography. An analgesic therapy was prescribed. Gravida 1 and parity 0. Planning on getting pregnancy. She had family history of endometriosis and not oncological family history. On gynecological examination there was a palpable pelvic firm mass of about $10 \mathrm{~cm}$ in size in the left iliac region, solid-elastic texture with tenderness and displacement of the uterus to the right.

Ultrasonography demonstrated a left ovarian cystic mass $(12.0 \times 8.7 \mathrm{~cm})$ with hyper-attenuated solid portions, thickened and with single loci of blood flow at CDI. The level of tumor markers HE4(63.72 pmol/L), CA 125(35.6U/mL), ROMA scores (23.97\%) indicated a high malignant ovarian lesions risk in premenopausal women. On workup there was not revealed a prevalence of tumor.

On laparoscopic surgical exploration the findings were left ovarian with adhesions, presented cystic mass measuring $11.5 \times 10.0 \mathrm{~cm}$; uterosacral ligaments and broad ligaments of the uterus with multiple endometriotic nodules from 0.5 to $1.2 \mathrm{~cm}$. Laparoscopic left ovarian cystectomy, excision and ablation of endometriotic nodules, endometrial biopsy was performed. Postoperative was uncomplicated.

Figure 1: Endometrioid borderline tumor, H\&E sections, 20x EE (a); expression of ER, 20x EE (b); Ki-67, 20x EE (c); BAF250a, $40 x \operatorname{EE}(d)$. 
Pathological findings presented both EBT with no invasion and endometriosis within this ovarian tumor. Microscopically, low papillary lesions with squamous morules were detected as well as focal atrophic glandular linings with polymorphic cells. Nuclear stratification was noted, but no invasive growth was apparent. Typical endometriosis lesions were present. Immunohistochemical studies demonstrated positive for estrogen receptor (ER), progesterone receptor (PR), Epithelial Membrane Antigen (EMA), negative for p53, alpha-inhibin, Wilms tumor gene (WT1). Ki67 labeling index was 8\%. Loss BAF250a (encoded by ARID1A) expression in EBT and endometriotic tissue did not observe (Figures 1a-1d).
Final diagnosis was ovarian endometrioid borderline tumor stage IA.

The patient had been taking Dienogest $2 \mathrm{mg}$ once daily treatment. The follow-up every three months within 36 months showed no evidence of disease recurrence. To date, this woman aged 29 years has a regular menstruation, no pelvic pain and does not plan a pregnancy in near future.

\section{Conclusion}

Therefore, this report implies association between endometriosis and EBT. Histological and IHC establish a diagnosis EBT and optimize surgical treatment in reproductive aged women, as well as preserve fertility. 